\title{
EL BENEFICIO ECLESIÁSTICO EN LA CIUDAD DE VALENCIA (PRIMERA MITAD DEL SIGLO XIV)
}

\author{
MARÍA JOSÉ CARBONELL BORIA \\ Universitat de València
}

\section{SUMARIO}

I. Patronos y beneficiados.- II. Beneficios documentados: A) Catedral B) Parroquias de la ciudad. C) Conventos de la ciudad. D) Hospitales de la ciudad

Los beneficios eclesiásticos de la primera mitad del s. XIV² en la diócesis valentina fueron fundados generalmente por familias nobles y las que aspiraban a serlo - en función de sus riquezas- aunque también encontramos fundaciones realizadas por eclesiásticos, principalmente obispos, canónigos y antiguos rectores de iglesias.

La mayoria de los beneficios se fundaban a través de testamentos, dotándolos de rentas pertinentes según el número de beneficiados que designaban. La cuantía de dichas rentas estaba supeditada a los deseos del fundador - no siempre respetados por sus herederos - y sus posibilidades económicas.

El presente trabajo se ha beneficiado, parcialmente. de la ayuda concedida por el Ministerio de Educación y Ciencia. DGICYT (PS95-0122) al proyecto de investigación: Escribir la sociedad. Cultura escrita y memoria histórica de la Comunidad Valenciana (1238-1474).

'Una primera aproximación a este tema para nuestra diócesis fue realizada por: José Sanchis Sivera. La Catedral de Valencia. Guía histórica y artística. Valencia. 1909. pp. 487 507: ID. La diócesis vialentina. Estudios históricos. Valencia. 1920; y por BARÓN DE SAN Petrilioo. Las Capillas Parrocquiales. Sirs blasones y patromos. "Anales del Centro de Cultura Valenciana" III (1942). pp. 103-114: IV (1943-44) pp. 9-69 y 145: V (1944). pp. 1-121 y 211

"Anuario de Lstudies Medievales", 28 (1998) 
En cuanto a los motivos de la fundación, el que más frecuentemente justificó la creación de un beneficio fue, sin lugar a dudas, el deseo de conseguir la salvación del alma del fundador, así como la remisión de sus pecados. Este motivo era el expuesto cuando dicha fundación venía contenida en un testamento.

Otras motivaciones ${ }^{3}$, aunque inferiores en número fueron: el cumplimiento de promesas realizadas, cómo acción de gracias por favores recibidos, salvaguarda del cumplimiento de los "oficios divinos" en zonas poco habitadas o de repoblación reciente, interés del fundador en asegurarse los "servicios particulares" de un clérigo -es decir, tener siempre un sacerdote a su disposición-, y finalmente ayuda al mantenimiento de clérigos pobres.

Por lo que respecta a las advocaciones ${ }^{4}$, el nombre del fundador fue uno de los factores que más influyó a la hora de dotar de invocación un beneficio, pues vemos que en la mayoría de los casos coinciden.

Otros factores que influirán en la elección de advocación para un beneficio son: el Santo patrono de la iglesia donde se funda el beneficio, el titular del altar o capilla donde se establece, el Santo o beato por el que siente devoción el fundador, y por último las corrientes de "moda" que potencian la veneración de un Santo concreto en determinadas épocas. En consecuencia, las advocaciones aparecidas en la ciudad de Valencia son muy similares a las de otras ciudades y diócesis de la antigua Corona de Aragón ${ }^{5}$. Aún así queremos destacar la presencia de la dedicada al Corpus Christi, por ser casi inexistente en el periodo que analizamos, al no haberse dado todavía la normativa a seguir respecto a su culto y veneración.

Este tipo de motivaciones al igual que la anterior, se mantendrợn incluso en época moderna. Cir: María Milagros CÁRCEL. ORTí. La dióccesis de Valencia y sus beneficiados (15011538). Valencia, 1980. Tesis doctoral mecanografiada.

${ }^{4}$ Respecto a lo que sucede en la archidiócesis de Valencia dos siglos más tarde Cfr: María Milagros Carcel. OrTi. Adrocaciones religiosas y omomaistica en la diócesis de Valencia (siglo $X V I)$. "Medievalia". 10 (1992) pp. 83-113.

SCfr. Joan Roselló LITERAS. Registra collationum ecclesie Maioricensis. "Fontes Rerum Balearium" 1 (1977), pp. 113-256; 2 (1978), pp. 81-160, 293-324, 517-548; 3 (1979-80) pp. 49-80; J.N. HillgarTH-G. Sil.ANO, The register "Notule Commumium" 14 of de Diocese of Barcelona (1345-1348), Toronto, 1983; Emilio SÁEZ. Adrocaciones religiosas en la Barcelona altomedieval (siglos IX-XIII), Barcelona, 1976. 


\section{PATRONOS Y BENEFICIADOS}

El fundador del beneficio es el que en primer lugar ostenta el título de patrono del mismo, reservándose el derecho de patronato. Este derecho quedaba adscrito a la propia familia con arreglo al orden de sucesión establecido, en el que se contemplaba, en el caso de extinción de la sucesión directa, la rama colateral a la que correspondería heredar el patronato.

Aunque el caso expuesto representa la tónica general seguida para el beneficio eclesiástico, vemos como en el periodo de pontificado de Ramón Gastón aparecen ya alguna excepciones:

El fundador, e incluso el colador, cuando aquél no disponía lo contrario, podía conceder el derecho de patronato a instituciones o corporaciones municipales - jurados de una ciudad, abad de un monasterio, etc.- de forma que dicho derecho no se hallaba sujeto a la línea sucesoria de una determinada persona, sino que dependía de las personas que en cada momento formasen la corporación designada.

Por otro lado, el prelado podía destituir o relevar de su cargo al patrono que no defendiera adecuadamente los intereses de su beneficio. En este caso el derecho de patronato pasaba al inmediato sucesor en la línea familiar, o si la destitución era temporal, al rector de la parroquia en donde se hallaba establecido el beneficio, e incluso a otros beneficiados de la misma parroquia cuya competencia estaba suficientemente probada.

Por lo que respecta a los beneficiados, vemos como la mayoría de los citados en nuestras fuentes han alcanzado ya el grado del presbiterato, e incluso algunos la dignidad de canónigos, pero no sucede así en todos los casos, pues también les son conferidos beneficios a: clérigos tonsurados $-38,9 \%$, clérigos que aún no han recibido las IIII minores ordines $-1,1 \%-, o$ también niños y jovenes cuya corta edad obliga al obispo a nombrar unn tutor y a la vez administrador de los bienes del beneficio $-0,5 \%-$. 


\section{BENEFICIOS DOCUMENTADOS}

Se presentan aquí, de forma sistematizada, los datos aportados por la documentación consultada ${ }^{6}$, ordenados alfabéticamente, empezando por la Catedral de Valencia, seguido de las restantes parroquias de dicha ciudad.

En cada beneficio indicamos: primero su advocación, que será la pauta que nos marca el orden alfabético seguido para su ordenación, después el nombre ${ }^{7}$ del fundador $y$ la fecha en que fue instituido dicho beneficio $y$, finalmente las fechas en que fue colado a distintos beneficiados, con los nombres de los mismos. Hay que indicar que además de por el criterio alfabético la ordenación de los beneficios está condicionada por el número de datos que conocemos del mismo, apareciendo siempre en primer lugar los beneficios con referencias más completas y acabando con los que tienen tan pocos datos que son dificilmente identificables o localizables.

\section{A) Catedral}

Capilla de la beata Catalina Tomás, antes Santa Lucia

1. Advocación: Santa Lucia. Fundado por Pere Pérez de Tarragona, canónigo de Valencia, en su último testamento, el 21 de noviembre de 1299, ante el notario Giner Rabaça. El 9 de Junio de 1346 dicho beneficio fue colado a Arnau de Palomar, vacante tras la muerte de Andreu Morell ${ }^{8}$.

2. Advoc.: Santa Lucia. Fundado por Arnau Çafont (de Fonte) en su último testamento, el 18 de octubre de 1315, ante el notario Pere Ferrer"

Capilla de la beata Inés de Benigamin, antes Santa Eulalia.

1. Advoc.: Santa Eulalia. Fundado por Guillem Julià, portero del rey de Aragón, el 8 de octubre de 1311 ante el notario Guillem Vernet. El 23

\footnotetext{
"Archivo Metropolitano de Valencia (AMV). Registro General de Colaciones 1316-1400. Sece. I. I:ondo III. Carpeta 1: AMV. Liber Collationum annorum MCCCXXXXVIMCCCXXXXVII. Sece I. Fondo III. Carpeta 2.: Archivo de la Catedral de Valencia (ACV). Pergaiminos 5048.

${ }^{7}$ Hemos respetado los nombres de todos los personajes tal y como aparecen en la documentación. $29 \mathrm{v}$.

${ }^{8}$ AMV, Secc. I, Fondo III. Carpeta 1, fol. 43; AMV. Secc. I, Fondo III. Carpeta 2, fol.

"AMV. Secc. I, Fondo III, Carpeta 1, fol. 1.
} 
de octubre de 1337, el beneficio es concedido a Hug Pellicer, vacante tras la renuncia de Guillem Pellicer ${ }^{10}$.

Capilla del beato Gaspar Bono, antes de San Nicolás obispo.

1. Advoc.: San Nicolás obispo. Fundado por Berenguer de Ripóll en su último testamento el 22 de abril de 1290 ante el notario Guillem Vernet ${ }^{11}$. Esta fundación no se hizo efectiva hasta el 30 de diciembre de $1328^{12}$. El 27 de noviembre de 1337 el beneficio es concedido a Bernat Garrigosa, vacante tras la renuncia de Joan Pere de Ripoll ${ }^{13}$. El 7 de enero de 1340, pasará a Bertomeu Boscà a causa de la muerte del dicho Bernat de Garrigosa $^{14}$.

\section{Capilla del Espíritu Santo (no existe actualmente)}

1. Advoc.: Espíritu Santo. Fundado por Nicolás de Hungria, canónigo, en su último testamento, el 28 de mayo de 1274, ante el notario Tomás Díez ${ }^{15}$. El 7 de mayo de 1334, el beneficio es concedido a Guillem Bosc, vacante tras la muerte de Felip Çavila ${ }^{16}$.

2. Advoc.: Espíritu Santo. Fundado por Ramona, mujer de Ramón Voltaràs en su último testamento. El 2 de junio de 1341 es concedido a Berenguer Bonell ${ }^{17}$.

Capilla de Ntra. Señora contra la Peste. Antes Sta. Catalina.

1. Advoc.: S. Andrés apóstol. Fundado por Pere d'Esplugues, arcediano de Alcira, en su testamento el 14 de mayo de 1337 ante el notario Arnau Ferrer. El 9 de diciembre de 1337 fue concedido a Pere de Manresa $^{18}$.

\footnotetext{
${ }^{10}$ AMV. Secc. I, Fondo III. Carpeta 1. fols. 73, 74r-v. 129v.

"José Sanchis Sivera, La catedral de Valencia. Guía histórica y artística. Valencia, 1909. p. 498.

"AMV. Secc. I. Fondo III. carpeta 1. fol. 10v.

"AMV. Secc. I. Fondo III. carpeta 1. fol. 10v.

${ }^{14}$ AMV, Secc. I. Fondo III, carpeta 1. fol. 12.

${ }^{15}$ José Sanchis Sivera, op. cit. p. 503.

${ }^{10} \mathrm{AMV}$, Secc. I, Fondo III, carpeta 1, fol. 4v.

${ }^{17} \mathrm{AMV}$, Secc. I, Fondo III, carpeta 1, fol. 26v.

${ }^{18}$ AMV. Secc. I, Fondo III, carpeta 1, fol. 10v.
} 
2. Advoc.: Sta. Catalina mártir. Fundado por Bernat de Canet, arcediano y canónigo, en su último testamento el 15 de junio de 1297, ante el notario Guillem Vernet. El 5 de enero de 1345, fue concedido a Estebe Roig, vacante tras la renuncia de su antecesor ${ }^{19}$.

Capilla de Ntra. Señora del Puig. Antes Santa Margarita.

1. Advoc.: Santa Margarita. Fundado por Jaume Ça Roca, deán de Valencia y después obispo de Huesca, el 9 de mayo de $1270^{20}$. El 30 de junio de 1334 es colado a Aparici Galindo vacante tras la muerte de Pere Sanç².

\section{Capilla de San Antonio}

1. Advoc.: San Juan Bautista. Fundado por Adam de Paterna ${ }^{22}$. El 16 de julio de 1345, es concedido a Llop Sanç d'Esquerra, vacante tras la muerte de Guillem Bonell ${ }^{23}$.

\section{Capilla de San Antonio Abad}

1. Advoc.: San Martín obispo. Fundado el 15 de septiembre de 1338 por Martí del Castellar, ante el notario Joan Monçó. El 26 de octubre de 1338, es concedido a Gil Pere ${ }^{24}$.

Capilla de San Antonio de Padua. Antes San Lorenzo.

1. Advoc.: San Lorenzo. Fundada por Pere de Ripoll ${ }^{25}$ en su testamento, el 9 de agosto de 1316, ante el notario Jaume de Miralles. El 2 de diciembre de 1337, es concedido a Joan Pere de Ripoll ${ }^{26}$. El 1 de diciembre de 1338 , renuncia a dicho beneficio Berenguer Ripoll ${ }^{27}$.

\footnotetext{
${ }^{10}$ AMV. Secc. I, Fondo III, carpeta 1, fol. 38v.

20)Jaume Ça Roca fundará dos beneficios en esta fecha y lugar.

"AMV. Secc. I. Fondo III. carpeta 1. fol. 5.

$\because A M V$. Secc. I. Fondo III. carpeta 1. fol. 13v.

${ }^{23}$ AMV. Secc. I, Fondo III, carpeta 1, fol. 41.

${ }^{24}$ AMV. Secc. I, Fondo III, carpeta 1, fol. 12v

${ }^{25} \mathrm{AMV}$. Secc. I, Fondo III, carpeta 1, fol. $11 \mathrm{v}$

"AMV. Secc. I, Fondo III, carpeta 1, fol. 9v.

${ }^{27}$ AMV. Secc. I, Fondo III, carpeta 1, fol. 12v.
} 
Capilla de San Dimas. Antes de la Espina.

1. Advoc.: Espina de la Corona del Señor. Fundado por el Cabildo de Valencia con los bienes de Arnau de Riusech, deán de la catedral. El 3 de noviembre de 1329, es concedido a Joan Carbonell, por permuta con Bernat de Bas ${ }^{28}$. El 14 de marzo de 1336, pasa a Bernat Bolas, a causa de la permuta habida con Ponç Soler ${ }^{29}$.

\section{Capilla de San Felipe Neri. Antes San Lucas.}

1. Advoc.: San Bartolomé. Fundado por Ramón d'Escorna, secretario del rey, en su testamento ante el notario Pere Martí, el 10 de febrero de 1291. El 31 de agosto de 1338, es concedido a Miquel Carví, vacante tras la muerte de Nicolau Conte ${ }^{30}$.

2. Advoc.: San Lucas. Fundado por Ramón d'Escorna, secretario del rey, en su testamento del 10 de febrero de 1291. El 27 de noviembre de 1335, es concedido a Nicolau Joan, vacante tras la muerte de Ramón Roger $^{31}$. El 10 de septiembre de 1338, pasa a Guillem Çavila ${ }^{32}$.

3. Advoc.: San Lucas. Fundado por Francesca Escorna, mujer de Pere Eximen de Borriol, en su testamento el 15 de mayo de 1333 ; ante el notario Andreu d'Espígol. El 6 de julio de 1334, es concedidó a Domènec Merino ${ }^{33}$.

4. Advoc.: San Lucas. Fundado por Joan Escorna, señor de Olocau, en su testamento del 23 de agosto de 1338, ante el notario Pere Fraga. El 10 de septiembre de 1338, es concedido a Guillem Çavila ${ }^{34}$.

\section{Capilla de San Gregorio.}

1. Advoc.: San Gregorio. Fundada por Ramón de Botçènit el 28 de mayo de 1310, ante el notario Ramón Escamarch. El 4 de octubre de 1334, es concedido a Bernat Reus, vacante tras la muerte de Ramón Ferrero ${ }^{35}$.

\footnotetext{
${ }^{28}$ AMV. Secc. I, Fondo III, carpeta 1, fol. 4.

${ }^{29} \mathrm{AMV}$. Secc. I, Fondo III, carpeta 1, fol. 17v.

${ }^{30}$ AMV, Secc. I, Fondo. III, carpeta 1, fol. 12.

${ }^{31}$ AMV, Secc. I, Fondo III, carpeta 1, fol. 16.

${ }^{32}$ AMV, Secc. I, Fondo III, carpeta 1, fol. 12.

${ }^{33} \mathrm{AMV}$, Secc. I, Fondo III, carpeta 1, fol. 5.

${ }^{34}$ AMV, Secc. I, Fondo III, carpeta 1, fol. 12.

${ }^{35}$ AMV. Secc. I, Fondo III, carpeta 1, fol. 5v; José Sanchis Sivera, op. cit. p. 318.
} 


\section{Capilla de San Honorato.}

1. Advoc.: San Honorato. Fundado por Guillem Daviu, canónigo, el 6 de abril de 1331, ante el notario Ramón Ferrer. El 13 de febrero de 1338, es concedido a Joan Olmeda ${ }^{36}$.

\section{Capilla de San Jaime.}

1. Advoc.: San Jaime, Fundada por el rey Jaime el 28 de enero de 1246, ante el notario Guillem Escrivà. El 8 de marzo de 1345 es concedido a Tomás Bonet ${ }^{37}$.

Capilla de San Juan Evangelista (no existe)

1. Advoc.: San Juan Evangelista. Fundada por Ramón de Montanyana, arcediano de Tarancón, el 15 de abril de $1316^{38}$. El 14 de junio de 1337 es concedido a Pere Pasqual, vacante tras la muerte de Oliver de Jaca $^{39}$

Capilla de San Juan de Ribera.

1. Advoc.: San Salvador. Fundado por Bernat Dego. El 10 de julio de 1335 es concedido a Jaume Bonastre a causa de la permuta habida con Jaume Vilagut ${ }^{40}$.

Capilla de San Luis obispo, antes San Bartolomé.

1. Advoc.: San Bartolomé. Fundado por Bernat Clapers en su testamento el 18 de septiembre de 1311. En 1329 es concedido a Guillem d'En Clapers, vacante tras la muerte de Berenguer Maciä ${ }^{41}$. El 26 de noviembre de 1334 , es concedido a Guillem des Clapers, tras la renuncia de Berenguer Belloc ${ }^{42}$. El 15 de enero de 1343 pasa a Guillem d'En Clapers, tras la renuncia del anterior, Guillem des Clapers ${ }^{43}$.

\footnotetext{
${ }^{36}$ AMV, Secc. I, Fondo III, carpeta 1, fol. 10.

${ }^{37}$ AMV. Secc. I, Fondo III, carpeta 1, fol. 40.

${ }^{38}$ ACV, Pergaminos, 5.048.

${ }^{39}$ AMV, Secc. I, Fondo III, carpeta 1, fol. 8v.

${ }^{40}$ AMV. Secc. I, Fondo III, carpeta 1, fol. 16.

"AMV, Secc. I, Fondo III, carpeta 1, fol. 4.

${ }^{42}$ AMV, Secc. I, Fondo III, carpeta 1, fol. 34v.

${ }^{43}$ AMV, Secc. I, Fondo III, carpeta 1, fol. 32v.
} 


\section{Capilla de San Mateo.}

1. Advoc.: San Miguel. Fundada por Beltrán de Terol, canónigo, el 4 de noviembre de 1256 , en su testamento ante el notario Domènec de $\mathrm{Jaca}^{44}$.

\section{Capilla de San Miguel.}

1. Advoc.: San Miguel Arcángel. Fundado por Ramón Gastón, obispo de Valencia el 29 de abril de $1324^{45}$. El 15 de febrero de 1336, es concedida a Ramón Gastó, vacante tras la muerte de Tomás Pujades ${ }^{46}$.

2. Advoc.: San Miguel, fundado por Ramón Gastón, obispo de Valencia el 29 de abril de 1324, ante el notario Ramón Serret ${ }^{47}$. El 21 de diciembre de 1337, es concedido a Arnau Gastó, tras la renuncia de Macià Alentorn $^{48}$.

Capilla de San Pedro. (no existe).

1. Advoc.: San Pedro. Fundado por Brunissèn de Montagut, mujer de Roderic de Figueroles, el 12 de julio de 1317. El 19 de septiembre de 1339, es concedido a Guillem Pinós, vacante tras la muerte de Domènec Martí $^{49}$.

Capilla de la "parroquia" de San Pedro. Antes San Luis.

1. Advoc.: San Luis. Fundado por Pedro de Urrea, obispo dê Huesca y señor de Canals, el 21 de diciembre de $1329^{50}$. En 1329 es concedido a J. P. Ferran ${ }^{51}$. El 23 de enero de 1343, es concedido a Tomás del Castellaar, vacante tras la muerte de Bernat Buada ${ }^{52}$.

\footnotetext{
${ }^{44}$ AMV, Secc. I, Fondo III, carpeta 1, fol. 19.

${ }^{45}$ AMV, Secc. I, Fondo III, carpeta 1, fol. 36v.

${ }^{46} \mathrm{AMV}$, Secc. 1, Fondo III, carpeta 1, fol. 16v.

${ }^{47}$ AMV, Secc. I, Fondo III, carpeta 1, fol. 36v.

${ }^{48} \mathrm{AMV}$, Secc. I, Fondo III, carpeta 1, fol. 11.

${ }^{49} \mathrm{AMV}$, Secc. I, Fondo III, carpeta 1, fol. 6.

${ }^{50}$ Pedro de Urrea funda en esta capilla cuatro beneficios el mismo día.

${ }^{51}$ AMV, Secc. I, Fondo III, carpeta 1, fol. 4.

${ }^{52}$ AMV, Secc. I, Fondo III, carpeta 1, fol. 39.
} 
2. Advoc.: - Fundada por García Pere de Biel. El 9 de octubre de 1329, vacante tras la muerte de Berenguer Gavet, fue colado a Ramón Vidal $^{53}$.

\section{Capilla de San Vicente.}

1. Advoc.: San Vicente mártir. Fundado por Pere Melet, natural de Vic y ciudadano de Valencia, en su testamento del 26 de septiembre de 1240. Esta institución no se hizo efectiva hasta el 25 de julio de 1277. El 9 de octubre de 1340 , es concedido a Guillem Melet, vacante tras la muerte de Pere Melet ${ }^{54}$. El 10 de octubre del mismo año, pasa a Jaume deç Vilar, a causa de la renuncia de Guillem Melet ${ }^{55}$.

2. Advoc.: San Vicente mártir. Fundado por Pere Melet, beneficiado en la catedral de Valencia, en su testamento del 8 de agosto de 1340. El 2 de octubre de 1340, es concedido a Jaume Marsicler ${ }^{56}$.

Capilla de San Vicent Ferrer. Antes del "Corpus Christi".

1. Advoc.: "Corpus Christi". Fundado por Pere Costa, arcediano de Játiva y canónigo de Valencia, en su testamento del 4 de agosto de 1320, ante el notario Guillem Gaucelino (de Narbona). El 1 de agosto de 1341, es concedido a Berenguer de Benasch, tras la renuncia de Pere $\mathrm{Mir}^{57}$.

\section{Capilla de San Vicente Ferrer. Antes de Todos los Santos.}

1. Advoc.: Pavordía de la Almoina. Fundado por Ramón Despont, obispo de Valencia, el 24 de mayo de $1303^{58}$. El 5 de febrero de 1334, fue concedido a Pere de Culadell, a causa de la permuta habida con Jaume Exarc $^{59}$.

\footnotetext{
${ }^{53} \mathrm{AMV}$, Secc. I, Fondo III, carpeta 1, fol. 3v.

${ }^{54} \mathrm{AMV}$, Secc. I, Fondo III, carpeta 1, fol. 22r-v.

${ }^{55} \mathrm{AMV}$, Secc. I, Fondo III, carpeta 1, fol. 22v.

${ }^{56} \mathrm{AMV}$, Secc. I, Fondo III, carpeta 1, fol. 22.

${ }^{57} \mathrm{AMV}$, Secc. I, Fondo III, carpeta 1, fol. 41

${ }^{58}$ Ramón Despont fundará dos beneficios en esta fecha y lugar y otros dos el 18 de marzo

${ }^{59} \mathrm{AMV}$, Secc. I, Fondo III, carpeta 1, fol. 32v
} de 1306. 
Capilla de San Vicente mártir. Antes San Dionisio.

1. Advoc.: San Dionisio. Fundado por Jaume de Pertusa, chantre y canónigo de la Catedral, el 18 de julio de $1340^{60}$.

2. Advoc.: San Vicente mártir, fundado por Vicent de Sant Vicent, canónigo, el 25 de octubre de 1272, ante el notario Ramón Massana. El 7 de octubre de 1341, es concedido a Guillem de Sant Vicent ${ }^{60}$.

Capilla de Santa Ana. (No existe).

1. Advoc.: Santa Ana. Fundado por Francesc de Luna y su mujer Margarita Escrivà, el 23 de agosto de 1343, ante el notario Bernat deç Cortell. El 10 de febrero de 1334, es concedido a Francesc de Jaca ${ }^{62}$.

2. Advoc.: Santa Ana. Fundado por Domènec Mateu, canónigo, el 21 de mayo de $1305^{63}$. El 18 de abril de 1317, es concedido a Domènec de Borja, rector de Pego, a causa de la permuta habida con Pere Martí64. El 14 de septiembre de 1334, es colado a Pasqual, tras la renuncia de su antecesor Antoni de Alpicat ${ }^{65}$. El 24 de octubre de 1335, pasa de nuevo a Antoni Alpicat, a causa de la permuta habida con Bernat Alpicat ${ }^{66}$.

Capilla de Santa Catalina mártir. Antes de Santo Domingo.

1. Advoc.: Santo Domingo. Fundado por Guillem de l'Om el 24 de noviembre de 326 ante el notario Jaume Miralles. El 22 de febrero de 1345 es concedido a Francesc Marrades, tras la privación de Pere de Iusa ${ }^{67}$.

\section{Capilla/Altar de Santa Lucía.}

1. Fundado por Octavià, canónigo de Valencia. El 2 de junio de 1346 es concedido a Guillem Bonpar, vacante tras la muerte de Domènec de la Abadia ${ }^{68}$.

\footnotetext{
${ }^{60} \mathrm{AMV}$, Secc. I, Fondo III, carpeta 1, fol. 21.

${ }^{61} \mathrm{AMV}$, Secc. I, Fondo III, carpeta 1, fol. 27v.

${ }^{62} \mathrm{AMV}$, Secc. I, Fondo III, carpeta 1, fol. 39.

${ }^{6.3}$ Domènec Mateu fundó este mismo día dos beneficios en dicha capilla.

${ }^{6+}$ AMV, Secc. I, Fondo III, carpeta 1. fol. 21v.

${ }^{65}$ AMV. Secc. I, Fondo III, carpeta 1, fol. 21v.

${ }^{66} \mathrm{AMV}$, Secc. I, Fondo III, carpeta 1, fol. 16.

${ }^{67} \mathrm{AMV}$. Secc. I, Fondo III, carpeta 1, fol. 39v.

${ }^{68} \mathrm{AMV}$. Secc. I, Fondo III, carpeta 2, fol. 28r-v.
} 
Capilla de Santa María Magdalena (No existe).

1. Advoc.: Santa María Magdalena. Fundada por Gisbert, obispo de Valencia, en su último testamento, el 7 de marzo de 1288, ante el notario Giner Rabasa. El 20 de septiembre de 1334 es concedido a Arnau Gastó ${ }^{69}$. El 21 de diciembre de 1337 pasa a Pere Pinyana tras la renuncia del dicho Arnau Gastó ${ }^{70}$.

2. Advoc.: Santa María Magdalena. Fundado por Pontilià Garriga, arcediano de Játiva y canónigo de Valencia, en su testamento del 28 de agosto de 1310, ante el notario Jaume Martí. El 23 de enero de 1339 es concedido a Bernat Berenguer ${ }^{71}$.

Capilla de Santa Marta (No existe).

1. Advoc.: Santa Marta. Fundado por Pere Llop el 30 de agosto de 1307. El 16 de abril de 1329 es concedido a Pere Agramunt vacante tras la muerte de Martí Garcés ${ }^{72}$.

Capilla de Santa Úrsula y las once mil Vírgenes (No existe).

1. Advoc.: Once mil Vírgenes. Fundado por Francesc de Montpalau es su testamento del 13 de julio de 1334, ante el notario Jaume Ricart. El 25 de febrero de 1336 es concedido a Berenguer Desprats ${ }^{73}$.

Capilla del Santo Bulto. Antes de San Antonio.

1. Advoc.: San Antonio. Fundado por Gil Martí d'Entença, señor de Náquera, el 23 de junio de 1341. El mismo día de su fundación es concedido a Pere Roig ${ }^{74}$.

Capilla de Santo Tomás de Villanueva. Antes San Blas.

1. Advoc.: San Blas. Fundado por Ramón de Cardona y Bernarda su mujer el 11 de octubre de 1280 . El 29 de marzo de 1335 es concedido a

\footnotetext{
${ }^{69} \mathrm{AMV}$, Secc. I, Fondo III, carpeta 1, fol. 5.

${ }^{70} \mathrm{AMV}$, Secc. I, Fondo III, carpeta 1, fol. 11

${ }^{71} \mathrm{AMV}$, Secc. I, Fondo III, carpeta 1, fol. 11.

${ }^{72} \mathrm{AMV}$, Secc. I, Fondo III, carpeta 1, fol. 3.

${ }^{73}$ AMV, Secc. I, Fondo III, carpeta 1, fol. 17

${ }^{74} \mathrm{AMV}$, Secc. I, Fondo III, carpeta 1, fol. 26v.
} 
Bernat Bolas, vacante tras la muerte de Jaume de Sant Feliu ${ }^{75}$. El 14 de marzo de 1336, pasa a Ponç de Soler, a causa de la permuta habida con Bernat Bolas ${ }^{76}$.

2. Advoc.: San Blas. Fundado por Ramón Masó el 17 de abril de 1337, ante el notario Arnau de Casesvelles. El 26 de abril de 1316, es concedido a Bertomeu Piquer ${ }^{77}$.

Otras fundaciones en la Catedral de localización incierta.

1. Advoc.: "Corpus Christi"78. Fundado por Jaume deç Vilar. El 4 de septiembre de 1436, su beneficiado, Berenguer deç Vilar, consigue licencia para ausentarse de dicho beneficio ${ }^{79}$.

2. Advoc.: Jesucristo. Fundado por Andreu d'Albalat, obispo de Valencia. El 1 de septiembre de 1338 es concedido a Guillem Dalmau ${ }^{80}$. Tras su muerte, el 19 de febrero de 1342 pasa a Arnau Escrivà ${ }^{81}$. En diciembre de 1343 es colado a Pere Escrivà ${ }^{82}$.

3. Advoc.: Jesucristo. Fundado por Maimó Martínez. En 1339 es concedido a Guillem de Menarges, tras la privación de Guillem Agost ${ }^{83}$, su anterior beneficiado.

4. Advoc.: San Antonio ${ }^{84}$. Fundado por "magister" Martinum. El 30 de mayo de 1317 es concedido a Anneco Sanç, a causa de la permuta habida con Joan Gascós .

5. Advoc.: San Bernardo. Fundado por Bernat de Nuça. El 7 de diciembre de 1339 es colado a Bernat de Nuor ${ }^{86}$.

\footnotetext{
${ }^{75}$ AMV, Secc. I, Fondo III, carpeta 1, fol. 15.

${ }^{76} \mathrm{AMV}$. Secc. I, Fondo III. carpeta 1, fol. 17v.

${ }^{77}$ AMV, Secc. I, Fondo III, carpeta 1, fol. Iv.

${ }^{78}$ Adscrita posiblemente a la actual capilla de San Vicent Ferrer antes del "Corpus".

${ }^{79} \mathrm{AMV}$, Secc. I Fondo III, carpeta 2, fol. 82r-v.

${ }^{80} \mathrm{AMV}$, Secc. I, Fondo III, carpeta 1, fol. 12.

${ }^{81} \mathrm{AMV}$, Secc. I, Fondo III, carpeta 1, fol. 25v.

${ }^{82} \mathrm{AMV}$, Secc. I, Fondo III, carpeta 1, fol. 34v.

${ }^{83} \mathrm{AMV}$, Secc. I, Fondo III, carpeta 1, fol. $5 \mathrm{v}$.

${ }^{84} \mathrm{Adscrita}$ probablemente a la actual capilla de San Antonio.

${ }^{85} \mathrm{AMV}$, Secc. I, Fondo III, carpeta 1, fol. $2 \mathrm{v}$

${ }^{86}$ AMV. Secc. I, Fondo III, carpeta 1, fol. 6v.
} 
6. Advoc.: San Gregorio papa ${ }^{87}$. Fundado por Bernat Amalda. El 17 de abril de 1340 es concedido a Pere Gastó tras la renuncia de su antecesor Pere de la Abadía ${ }^{88}$. En 1341, pasará a Pere Pallarés ${ }^{89}$.

7. Advoc.: San Guillermo. Fundado por Guillem y Jaume Escrivà el 20 de marzo de 1257. En 1334 fue colado a fray García d'Alcàsser, de la Orden de la Santísima Trinidad ${ }^{90}$. El 29 de agosto de 1335, vacante tras la muerte de García d'Alcàsser, es concedido a fra Roland Vilaur ${ }^{91}$.

8. Advoc.: San Juan Bautista. Fundado por Peirona Escrivà y María Bata. El 30 de diciembre de 1342, vacante tras la muerte de Bernat deç Torrent, es concedido a Berenguer Colomer ${ }^{92}$.

9. Advoc.: San Nicolás ${ }^{93}$. Fundado por Constanza, mujer de Tovia d'Eslava. El 24 de marzo de 1334 es conferido a Guillem Fábrega ${ }^{94}$.

10. Advoc.: Santa Bárbara ${ }^{95}$. Fue fundado por una provisión del papa Clemente VI. El 27 de marzo de 1343 es concedida a Pere Ferrer, rector de Pina ${ }^{96}$.

11. Advoc.: Santa Lucía $^{97}$, el 17 de julio de 1346 es conferido a Jaume Arbocer, a causa de la permuta habida con Andreu David ${ }^{98}$. El 7 de septiembre de 1346 pasa a Pere Voltaràç

12. Advoc.: Santísima Virgen. Fundado por Bernat. El 23 de abril de 1334 es concedido a Guillem Alarich, vacante tras la muerte de Pere de Villalonga ${ }^{1(x)}$.

\footnotetext{
${ }^{87}$ Adscrita posiblemente a la actual capilla de San Gregorio.

${ }^{88}$ AMV. Secc. I, Fondo III, carpeta 1, fol. 20.

${ }^{89} \mathrm{AMV}$, Secc. I, Fondo III, carpeta 1, fol. 27v.

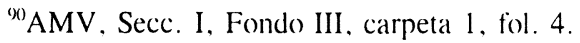

"AMV. Secc. I, Fondo III, carpeta 1, fol. 37v.

92AMV. Secc. I, Fondo III, carpeta 1, fol. 31v.

${ }^{93}$ Probablemente adscrita a la actual capilla del beato Gaspar Bono, antes de San Nicolás.

${ }^{94}$ AMV, Secc. I, Fondo III, carpeta 1, fol. 40v.

${ }^{25}$ Adscrito posiblemente a la actual capilla del Nacimiento, antes de Santa Bárbara.

${ }^{x}$ AMV, Secc. I, Fondo III, carpeta 1, fol. 34. Lucia.

${ }^{97} \mathrm{Con}$ probable adscripción a la actual capilla de la beata Catalina Tomás, antes de Santa $55 \mathrm{v}$.

${ }^{18}$ AMV, Secc. I, Fondo III. carpeta 1, fol. 43v; AMV, Secc. I, Fondo III, carpeta 2, fol.

"9MV, Secc. I, Fondo III, carpeta 2, fol. 82v

${ }^{1(x)}$ AMV, Secc. I, Fondo III, carpeta 1, fol. $4 \mathrm{v}$.
} 
13. Advoc.: - Fundado por Berenguer Canelles. En septiembre de 1334 es concedido a Jaume Melet ${ }^{101}$.

14. Advoc: - Fundado por Jaume Company. En octubre de 1342, vacante tras la muerte de su último poseedor, es concedido a Arnau Cerveró ${ }^{102}$.

15. Advoc. - Fundado por el Obispo y Cabildo con los bienes del canónigo Arnau de Celma. El 5 de agosto de 1338, vacante tras la muertè de Bernat Celon, es conferido a Martí Nebot ${ }^{103}$.

16. Advoc.: - Fundado por "Franavia", mujer de Ramón Berenguer. El 21 de marzo de 1339 es concedido a Pere Berenguer, tras la renuncia de Bernat Berenguer ${ }^{104}$. El 8 de marzo de 1345 pasará a Arnau Berenguer, al quedar vacante por el matrimonio del dicho Pere Berenguer ${ }^{105}$.

17. Advoc.:- Fundado por María, mujer de Ramón Berenguer. El 29 de junio de 1334, es concedido a Bernat Berenguer ${ }^{106}$.

18. Advoc.:- Fundado por Domènec y Pere Masó. El 6 de abril de 1337, vacante tras la muerte de Joan Gavaldà, es conferido a Ramón Bertrán $^{107}$.

19. Advoc.: - Fundado por Joan de Na Maura. El 19 de noviembre de 1316, es concedido a Ramón Andreu ${ }^{108}$.

\section{B) Parroquias de la ciudad}

\section{San Andrés}

1. Advoc.: San Juan Bautista y Evangelista. Fundado por Guillem Bosch. El 6 de junio de 1340 es conferido a Bertomeu Ripoll ${ }^{109}$.

\footnotetext{
${ }^{101}$ AMV, Secc. I. Fondo III, carpeta 1, fol. 14.

${ }^{102}$ AMV, Secc. I, Fondo III, carpeta 1, fol. 31.

${ }^{103} \mathrm{AMV}$, Secc. I, Fondo III, carpeta 1, fol. 11v.

${ }^{104}$ AMV, Secc. I, Fondo III, carpeta 1, fol. 11v.

${ }^{105}$ AMV, Secc. I, Fondo III, carpeta 1, fol. 40.

${ }^{106} \mathrm{AMV}$, Secc. I, Fondo III, carpeta 1, fol. 5.

${ }^{107}$ AMV, Secc. I, Fondo III, carpeta 1, fol. 8.

${ }^{108} \mathrm{AMV}$, Secc. I, Fondo III, carpeta 1, fol. 2.

${ }^{109} \mathrm{AMV}$, Secc. I, Fondo III, carpeta 1, fol. 20v.
} 
2. Advoc.: San Juan Bautista y Evangelista. Fundado por Maimó Çaplana ${ }^{110}$. El 8 de julio de 1341, vacante tras la muerte de Bertomeu deç Molins, es concedido a Andreu Çaplana"'I.

3. Advoc.: San Marcos. Fundado por Joan deç Camps. El 12 de octubre nos aparece la noticia de la muerte de su beneficiado Joan Boïl ${ }^{112}$.

4. Advoc.: - Fundado por María, mujer de Ramón Bages, el 28 de agosto de $1329^{113}$.

\section{San Bartolomé}

1. Advoc.: Santa Catalina. Fundado por María, mujer de Joan Ivanyes, en su testamento. El 7 de agosto de 1341 es concedido a Joan Ivanyes 114

2. Advoc.: - Fundado por Bertomeu de Solsona ${ }^{115}$. El 15 de julio de 1346 es concedido a Bernat Bertomeu, tras la renuncia de su anterior beneficiado Felip Serrano ${ }^{116}$.

3. Advoc.: - Fundado por Domènec de Rufes. El 14 de julio de 1336, vacante tras la muerte de Bernat Rufes, es concedido a Pere Rufes $^{117}$.

\section{San Esteban}

1. Advoc.: San Bartolomé y Santa Bárbara. Fundado por Bertomeu Barberà $^{118}$. El 6 de junio de 1335 es concedido a Francesc Ferrer ${ }^{119}$.

2. Advoc.: Santa María Virgen. Fundado por Ramón Poblet ${ }^{120}$.

\footnotetext{
${ }^{110} \mathrm{AMV}$, Secc. I, Fondo III, carpeta 1, fol. 20v.

"'AMV, Secc. I, Fondo III, carpeta 1, fol. 26v-27.

${ }^{112}$ AMV, Secc. I, Fondo III, carpeta 1, fol. 37v.

${ }^{113}$ AMV, Secc. I, Fondo III, carpeta 1, fol. 3v.

${ }^{114}$ AMV, Secc. I, Fondo III, carpeta 1, fol. 27v.

${ }^{115}$ AMV, Secc. I, Fondo III, carpeta 1, fol. 43v; AMV, Secc. I. Fondo III, carpeta 2, fol.

${ }^{116}$ AMV, Secc. I, Fondo III, carpeta 1, fol. 43v; AMV, Secc. I. Fondo III, carpeta 2, fol.

${ }^{117}$ AMV, Secc. I, Fondo III, carpeta 1, fol. 18.

${ }^{118}$ Adscrito posiblemente a la actual capilla de los Falcó.

${ }^{119}$ AMV. Secc. I. Fondo III. carpeta 1. fol. 15v.

${ }^{120}$ AMV, Secc. I, Fondo III, carpeta 1, fol. 26
} 53. $52 \mathrm{v}-53$. 
3. Advoc.: - Fundado por Berengaria, mujer de Martí del Castellar. El 1 de marzo de 1345, es concedido a Ramón Tarragona al quedar vacante tras la muerte de su antecesor ${ }^{121}$.

4. Advoc.: - Fundado por Guillem Martí. El 22 de noviembre de 1340 es conferido a Jaume Gil, tras la muerte de Aparici Gil ${ }^{122}$. El 28 de junio de 1334, al morir Jaume Gil pasa a Nicolau Bonfill' ${ }^{123}$.

5. Advoc.:- Fundado por Nicolau Valança. El 17 de junio de 1343 es concedido a Francesc Ferrer ${ }^{124}$.

\section{San Juan del Mercado}

1. Advoc.: Cátedra de San Pedro. Fundado por Guillem de la Porta. El 14 de mayo de 1341 es concedido a Bernat Pujol, tras la muerte de Martí Mateu $^{125}$.

2. Advoc.: "Corpus Christi". Fundado por María Pérez, mujer de Pere Arrufat ${ }^{126}$. El 15 de abril de 1334, vacante tras la muerte de Guillem Alleger, es concedido a Gil Alemany ${ }^{127}$.

3. Advoc.: San Antonio. Fundado por Pere Soler el 22 de enero de $1316^{128}$. El 27 de mayo de 1317, es presentado Berenguer Gomiza para acceder al beneficio ${ }^{129}$.

4. Advoc.: San Jaime Apóstol ${ }^{130}$. Fundado por Pere Ferrer, rector de dicha iglesia, el 7 de mayo de 1339.31. El 28 de enero de 1335, vacante tras la muerte de Garcia de Montsó, es concedido a Jaume Na Serra ${ }^{132}$. El 27 de octubre de 1340, es presentado Jaume de Montesa, para cubrir la

\footnotetext{
${ }^{121}$ AMV, Secc. I, Fondo III, carpeta 1, fol. 40.

1'2AMV, Secc. I, Fondo III, carpeta 1, fol. 23v.

123AMV. Secc. I. Fondo III, carpeta 1, fol. 37.

${ }^{124}$ AMV. Secc. I, Fondo III, carpeta 1, fol. 34.

${ }^{125}$ AMV, Secc. I, Fondo III, carpeta 1, fol. 26.

12"AMV. Secc. I, Fondo III, carpeta 1, fol. 21.

${ }^{127}$ AMV, Secc. I, Fondo III, carpeta 1, fol. 4.

${ }^{128}$ Archivo Conde de Orgaz. Fondo Crespí de Valldaura. ca/XI-I; Cfr. Vicente Pons ALós, Tesis doctoral mecanografiada. Valencia 1986.

${ }^{129}$ AMV, Secc. I, Fondo III, carpeta 1, fol. 2v.

${ }^{130} \mathrm{El}$ beneficio se hallaba adscrito al altar mayor.

${ }^{131}$ AMV, Secc. I, Fondo III, carpeta 1, fol. 5v.

"AMV. Secc. I, Fondo III, carpeta 1, fol. 14v.
} 
vacante dejada por la renuncia de su anterior beneficiado Jaume $\mathrm{Na}$ Serra ${ }^{133}$.

5. Advoc.: San Jorge. Fundado por Guillem Crespí. El 3 de noviembre de 1337 es concedido a Vicent Crespi ${ }^{134}$.

6. Advoc.: San Miguel. Fundado por Romea, mujer de Ramón L., el 27 de septiembre de $2326^{135}$. En octubre de 1326 es concedido a Eximén de Vayllo ${ }^{136}$.

7. Advoc.: Santa Lucia. Fundado por Domènec de Aiussa (d'Insa). El 8 de enero de 1345, es presentado Arnau Comes para cubrir la vacante habida tras la muerte del último beneficiado Francesc d'Aiussa ${ }^{137}$. El 9 de mayo de 1346, es definitivamente concedido a Pere Benencasa ${ }^{138}$.

8. Advoc.: Santísima Trinidad. Fundado por Guillem deç Vilar. El 16 de mayo de 1343 es concedido a Bertomeu Iborra ${ }^{139}$.

9. Advoc.: Todos los Santos. Fundado por Guillem Pineda. El 5 de julio de 1345 es concedido a Berenguer d'Estalric ${ }^{140}$.

10. Advoc.: - Fundado por Caterina, mujer de Gil Martínez d'Entenza, en su último testamento, el 20 de mayo de $1334^{141}$.

11. Advoc.: - Fundado por Ramón Oliver. El 10 de julio de 1342 es concedido a Bernat Montbalnch ${ }^{142}$.

12.- Advoc.: - Fundado también por Raimón Oliver. El 10 de julio de 1342 es concedido a Vicent Estella ${ }^{143}$.

\footnotetext{
${ }^{13.3}$ AMV, Secc. I, Fondo III, carpeta 1, fol. 23.

${ }^{134}$ AMV, Secc. I, Fondo III, curpeta 1, fol. 9v.

${ }^{135}$ AMV, Secc. I, Fondo III, carpeta 1, fol. 23.

${ }^{136} \mathrm{AMV}$, Secc. 1, Fondo III, carpeta 1, fol. 22v.

${ }^{137}$ AMV, Secc. I, Fondo III, carpeta 1, fol. 38v

${ }^{138}$ AMV, Secc. I, Fondo III, carpeta 1, fol. 43; AMV, Secc. I, Fondo III, carpeta 2, fol.

${ }^{139}$ AMV, Secc. I, Fondo III, carpeta 1, fol. 33v.

${ }^{1+1)}$ AMV, Secc. I, Fondo III, carpeta 1, fol. 41.

${ }^{1+1}$ AMV, Secc. I, Fondo III, carpeta 1, fol. 4v.

${ }^{142}$ AMV, Secc. I, Fondo III, carpeta 1, fol. 29v.

${ }^{14.3}$ AMV, Secc. I, Fondo III, carpeta 1, fol. 29v.
} $13 v$. 


\section{San Juan del Hospital}

1. Advoc.: Santa Catalina. Fundado por Sança de Alvar. El 1 de julio de 1340, vacante tras la muerte de García Llop, es concedido a Jaume de Llíria ${ }^{1+4}$.

2. Advoc.: Santa María Magdalena. Fundado por Arnau Llançol. El 9 de marzo de 1345 es concedido a Pere Sabata ${ }^{1+5}$.

3. Advoc.: Santa María Magdalena. Fundado por Saurina de Romaní. El 16 de abril de 1316 es concedido a Guillem Soler ${ }^{146}$. El 20 de agosto de 1340, vacante tras la muerte de Pere Gamell, es colado a Martí de la Cera ${ }^{1+7}$.

4. Advoc.: - Fundado por Barcelona, mujer de Berenguer Maçanet. El 3 de noviembre de 1338, vacante tras la muerte de García Corts, es concedido a Bernat Pujor ${ }^{1+8}$. El 24 de noviembre de 1346 pasa a Bernat Pucullull ${ }^{1+9}$.

5. Advoc.: - Fundado por Jaume de Romaní el 9 de diciembre de 1333. El 27 de mayo de 1335 es concedido a Pere Fiscall ${ }^{150}$.

6. Advoc.: - Fundado por Galcerana de Tous, mujer de Jaume de Tous, en su último testamento. El 5 de noviembre de 1338, es colado a Pere de Morera, vacante a consecuencia del matrimonio de su anterior beneficiado Miquel de Emena ${ }^{151}$.

7. Advoc.: - Fundado por Carmona de Vera, mujer de Gonçal Pere d'Ull. El 26 de abril de 1316 es concedido a Pere de Odena, con motivo de la permuta habida con Joan de Pulla ${ }^{152}$. El 9 de mayo de 1346 es conferido a fra Antoni Ça Guardia, vacante tras la muerte de fra Pere Alfons del $\operatorname{Rei}^{153}$.

\footnotetext{
${ }^{1+4}$ AMV. Secc. I. Fondo III. carpeta 1. fol. 20v.

${ }^{145}$ AMV. Secc. I. Fondo III. carpeta 1, fol. 40.

1.t'AMV. Secc. I. Fondo III. carpeta 1. fol. 2.

${ }^{1+17}$ AMV. Secc. I. Fondo III. carpeta 1. fol. 21.

${ }^{1+\mathrm{A}} \mathrm{AMV}$. Secc. I. Fondo III, carpeta 1. fol. 12v.

${ }^{1+19}$ AMV. Secc. I. Fondo III. carpeta 2. fol. 122r-v. 123.

${ }^{150}$ AMV. Secc. I. Fondo III, carpeta 1, fol. 15v.

${ }^{151}$ AMV, Secc. I, Fondo III, carpeta 1, fol. 12v.

${ }^{152}$ AMV. Secc. I, Fondo III, carpeta 1, fol. 1.

${ }^{153}$ AMV, Secc. I, Fondo III. carpeta 1, fol. 43; AMV. Secc. I, Fondo III. carpeta 2, fol.
} $11 v-12$. 


\section{Advoc.: - Fundado por Na Maçaneta ${ }^{154}$.}

\section{San Lorenzo}

1. Advoc.: Nuestra Señora "la Redonda". Fundado por el infante don Pedro, con los bienes de Pere Forés ${ }^{155}$, el 4 de junio de $1341^{156}$.

2. Advoc.: - Adscrito al altar mayor. Fundado por Marc de Soria, arcediano de Teruel. El 20 de noviembre de 1340, vacante tras la muerte de Pere Eiximenis, es concedido a Pere de Thever ${ }^{157}$.

3. Advoc.: - Fundado por Martí de Tovia, arcipreste de Teruel. El 31 de agosto de 1334 , vacante tras la muerte de Bernat de Biure, es colado a Bernat Maça ${ }^{158}$. El 25 de octubre de 1339, es conferido a Domènec Soler, vacante tras la muerte de Miquel de Miranda ${ }^{159}$. El 11 de septiembre de 1340 pasa a Guillem Contijoch, con motivo de la permuta habida con Domènec de Soler ${ }^{1(0)}$. El 11 de julio de 1342, es concedido a Bernat Perdinyà, a causa de una nueva permuta con el anterior ${ }^{161}$.

4. Advoc.: - El 2 de enero de 1336 es concedido a Guillem Gascó, vacante tras la muerte de Ramón Comalada ${ }^{162}$.

\section{San Martín}

1. Advoc.: Beata María. Fundado por Teresa, mujer de Jaume Despí. El 13 de marzo de 1339 es concedido a Alfons Cuadrat ${ }^{16.3}$.

2. Advoc.: Nuestra Señora. Fundado por Nicolau Vallterça. El 28 de mayo de 1334 es conferido a Ramón de Montblanch, tras la renuncia de Bernat Blanc ${ }^{164}$.

\footnotetext{
${ }^{154}$ AMV, Secc. I, Fondo III, carpeta 1, fol. 17v.

${ }^{155} \mathrm{AMV}$, Secc. I, Fondo III, carpeta 1, fol. 21.

${ }^{156}$ AMV. Secc. I, Fondo III, carpeta 1, fol. 26.

${ }^{157}$ AMV. Secc. I, Fondo III, carpeta 1, fol. 23.

${ }^{158}$ AMV. Secc. I, Fondo III, carpeta 1, fol. 5.

${ }^{159} \mathrm{AMV}$, Secc. I, Fondo III, carpeta 1, fol. 6v.

${ }^{1(x)}$ AMV, Secc. I, Fondo III, carpeta 1, fol. $21 \mathrm{v}$.

${ }^{161}$ AMV, Secc. I, Fondo III, carpeta 1, fol. 29v.

${ }^{162}$ AMV, Secc. I, Fondo III, carpeta 1, fol. 7.

${ }^{163} \mathrm{AMV}$, Secc. I, Fondo III, carpeta 1, fol. 11v.

${ }^{16+4}$ AMV, Secc. I, Fondo III, carpeta 1, fol. 36v.
} 
3.- Advoc.: - Fundado por Jauma, mujer de Berenguer Gisbert, en su último testamento. El 18 de noviembre de 1343 es concedido a Guillem Burguet ${ }^{165}$.

4. Advoc.: - Fundado por Guillem de Peralada. El 4 de octubre de 1334 , tras la renuncia de Jaume Puigalt, es concedido a Bernat Joan ${ }^{166}$.

\section{San Nicolás}

1. Advoc.: San Bernardo confesor. Fundado por Berenguer Suau. El 24 de julio de 1340 es concedido a Pere de Piella ${ }^{167}$. Gerarda $^{168}$.

2. Advoc.: Santa Eulalia. Fundado por Jaume Bavita y su mujer

3. Advoc.: - Fundado por Ramón de Moguer el 10 de mayo de 1329. El 23 de mayo de 1329 es presentado Pere Pellicer para ocupar dicho beneficio ${ }^{169}$.

4. Advoc.: - Fundado por Esclaramunda, mujer de Bertomeu de Pego ${ }^{171}$. El 3 de abril de 1329 es concedido a Mateu Pons, con motivo de la permuta habida con Ramón Forca ${ }^{17 !}$.

\section{San Salvador}

1. Advoc.: San Jorge. Fundado por Guillem (Berenguer) Maymó, alias Conill. El 26 de febrero de 1334 es concedido a Jaume Claramunt, alias Bonfill ${ }^{172}$. El 22 de febrero de 1345, pasa a Jaume Bonfill, con motivo de la renuncia del anterior beneficiado, el citado Jaume Claramunt ${ }^{173}$.

\footnotetext{
${ }^{11,5}$ AMV. Secc. 1. Fondo III, carpeta 1. fol. 34v.

Ios AMV. Secc. I. Fondo III. carpeta 1, fol. 14.

${ }^{167}$ AMV. Secc. I. Fondo III. carpeta 1. fol. 21.

${ }^{118}$ AMV. Secec. I. Fondo III. carpeta 1. fol. 32v.

${ }^{164}$ AMV. Secc. I. Fondo III. carpeta 1. fol. 3.

170'AMV. Secc. I. Fondo III. carpeta 1. fol. 2.

${ }^{171}$ AMV. Secc. I, Fondo III. carpeta 1. fol. 2v.

17'AMV. Secc. I. Fondo III. carpeta 1. fol. 33.

${ }^{173}$ AMV. Secc. I. Fondo III, carpeta 1. fol. 39v.
} 


\section{Santa Catalina}

1. Advoc.: Nuestra Señora de la Esperanza. Fundado por Domènec Aguiló el 14 de enero de $1304^{174}$. El 13 de agosto de 1346, fue concedido a Bernat de Cursa, vacante tras la muerte de Andreu Morell ${ }^{175}$.

2. Advoc.: Nuestra Señora y todos los Apóstoles. Fundado por Salvador Ric. El 21 de diciembre de 1343 es concedido a Arnau Moya ${ }^{176}$.

3. Advoc.: San Andrés. Fundado por Guillem Carbonell el 13 de mayo de 1346. El 29 de julio de 1346, es conferido a Joan Çavila ${ }^{177}$.

4. Advoc.: San Ildefonso. Fundado por Alfons Martí de Ça Morera. El 31 de agosto de 1335 es colado a Ramón Tarragona ${ }^{178}$. El 7 de febrero de 1337, tras la muerte de dicho Ramón Tarragona, pasa a Pere de Manresa $^{179}$.

5. Advoc.: San Ildefonso. Fundado también por Alfons Martí de Ça Morera el 26 de julio de 1339. En agosto de 1339 es concedido a Jaume deç Vilar ${ }^{180}$. El 23 de enero de 1341 pasa a Bernat Alegret, tras la renuncia del mencionado Jaume deç Vilar ${ }^{181}$.

6. Advoc.: San Miguel Arcángel ${ }^{182}$. Fundado por Berenguer Anglés. El 29 de agosto de 1337, es concedida a Pere Agulló ${ }^{183}$. El 6 de abril del mismo año pasa a Arnau Bru ${ }^{184}$.

\footnotetext{
${ }^{174}$ AMV. Secc. I. Fondo III, carpeta 1, fol. 44.

${ }^{175}$ AMV. Secc. I. Fondo III, carpeta 2, fol. 8v.

${ }^{170} \mathrm{AMV}$, Secc. I, Fondo III, carpeta 1, fol. 35.

${ }^{177} A M V$, Secc. I, Fondo III, carpeta 2. fol. 61r-v.

${ }^{178}$ AMV, Secc. I, Fondo III, carpeta 1, fol. 15v.

${ }^{179}$ AMV. Secc. I, Fondo III, carpeta 1, fol. 7 y 10v.

${ }^{180}$ AMV, Secc. I, Fondo III, carpeta 1. fol. 6.

${ }^{181}$ AMV. Secc. I. Fondo III. carpeta 1, fol. 23v.

182Adscrito a la actual capilla de los Claramunt, también llamada del Cristo y antiguamente de San Miguel y San Blas.

${ }^{18:}$ AMV, Secc. I, Fondo III. carpeta 1, fol. 9.

${ }^{18 .}$ AMV. Secc. I, Fondo III, carpeta 2, fol. 7r-v.
} 
7. Advoc.: Santa María. Fundado por Bernat Segarra el 19 de noviembre de $1346^{185}$. El 5 de diciembre del mismo año fue colado a Jaume Pere ${ }^{186}$.

8. Advoc.: - Fundado por Joan Cerveró ${ }^{187}$.

9. Advoc.: - Fundado por Arnau Constantí. El 16 de marzo de 1336 es concedido a Ramón Gostans ${ }^{188}$.

10. Advoc.: - Fundado por Bernat de Narbona y su mujer Estefanía. El 31 de julio de 1342 es concedido a Bernat del Mas ${ }^{189}$.

11. Advoc.: - Fundado por Guillem de Prat Boí. El 22 de marzo de 1340, vacante tras la muerte de Joan Llop, es concedido a Bernat de Prat Boi $^{191}$.

12. Advoc.: - Fundado por Pere de Segarra. El 23 de abril de 1341, es concedido a Arnau Filell ${ }^{191}$. El 1 de marzo de 1342, tras la renuncia del dicho Arnau, es colado a Pere Simeó ${ }^{192}$.

13. Advoc.: - Fundado por Jaume Soler ${ }^{193}$.

\section{Santa Cruz}

1. Advoc.: San Esteban. Fundado por Jaume Esteve. El 26 de agosto de 1336 es concedido a Esteve Marté ${ }^{144}$.

2. Advoc.: Santa Ana. El 26 de abril de 1316 su beneficiado, Miquel Montsó, presenta la renuncia a dicho beneficio. El 22 de julio del mismo año, reitera su renuncia ${ }^{195}$.

\footnotetext{
136v.

${ }^{185}$ AMV. Sece. I. Fondo III. carpeta 1. fol. 45: AMV. Secc. I. Fondo III. carpeta 2. foll

Ist AMV. Secc. I. Fondo III. carpeta 1, fol. 45: AMV. Secc. I. Fondo III. carpeta 2. fol 136r-v.

${ }^{187}$ AMV. Secc. I. Fondo III. carpeta 1, fol. 32v.

${ }^{18 x}$ AMV. Secc. I. Fondo III. carpeta 1, fol. 17.

${ }^{180}$ AMV. Secc. I. Fondo III. carpeta 1. fol. 29v.

${ }^{(x)} A M V$. Secc. I. Fondo III. carpeta 1. fol. 13v

"'AMV. Secc. I. Fondo III. carpeta 1, foll. 26.

"'AMV. Secc. I. Fondo III. carpeta 1, fol. 25v.

${ }^{19:}$ AMV. Secc. I, Fondo III. carpeta 1, fol. 43v.

${ }^{194}$ AMV. Secc. I, Fondo III, carpeta 1. fol. 18.

${ }^{105}$ AMV, Secc. I. Fondo III. carpeta 1. fol. Iv.
} 
Santa Maria del Olivar (hoy Monte Olivete)

1. Advoc.: Santa María Magdalena. Fundado por la madre de Ponç de Soler. El 7 de junio de 1341 tenemos noticia de que Bernat Monet regentaba el beneficio ${ }^{196}$.

\section{Grao (de Valencia)}

1. Advoc.: San Antonio confesor. Fundado por Berenguer Maimó y su mujer. El 21 de julio de 1334 es concedido a Jaume Bealeia ${ }^{197}$.

\section{Real (de Valencia)}

1. Advoc - Fundado por el infante Pere el 23 de agosto de $1329^{198}$. El 24 de mayo de 1334 es concedido a Bernat Navinal, vacante tras la muerte de Berenguer de Ripoll ${ }^{199}$.

\section{C) Conventos de la ciudad}

\section{De la Concepción (Orden de San Francisco)}

1. Advoc.: Santa Isabel. Fundado por Eximén Pere d'Oriz. En abril de 1316 es concedido a Joan de Pulla con motivo de la permuta habida con Pere Odena ${ }^{2(1)}$.

De San Julián (Orden de San Agustín)

1. Advoc.: San Julián. Fundado por Ramón Gascó. En 1339, vacante tras la muerte de Guillem Barreda, es concedido a Benet Garín ${ }^{201}$.

De San Vicente Mártir (Orden de la Merced)

1. Advoc.: San Mateo apóstol. Fundado por Aparici, portero de la reina de Aragón. El 7 de febrero es colado a Aparici Ganalor ${ }^{212}$. El 20 de febrero de 1336 es conferido a Arnau Buades. El 4 de marzo de 1336, pasa

\footnotetext{
${ }^{196}$ AMV, Secc. I, Fondo III, carpeta 1, fol. 26v

${ }^{197}$ AMV. Secc. I, Fondo III. carpeta 1. fol. 37.

${ }^{198}$ AMV. Secc. I. Fondo III. carpeta 1, fol. 3.

${ }^{199}$ AMV. Secc. I, Fondo III. carpeta 1, fol. 4v.

${ }^{200}$ AMV. Secc. I, Fondo III, carpeta 1. fol. 1.

2"1)AMV, Secc. I, Fondo III, carpeta 1, fol. 6.

212AMV. Secc. I, Fondo III, carpeta 1, fol. 2.
} 
a Jaume Beniader, con motivo de la permuta habida con el citado Arnau Buades $^{2013}$

2. Advoc.: - Fundado por Guillem de Jaca. En 1336 es concedido a Ramón Porta a causa de la permuta habida con Guillem Feliu ${ }^{204}$.

\section{De San Vicente Mártir (Orden de la Merced)}

1. Advoc.: - Fundado por Pere Eximén de Borriol en su testamento $^{205}$ del 13 de noviembre de $1341^{206}$. El 9 de agosto de 1345 es concedido a Pere d'Algarra, tras la renuncia de Berenguer de Benasch ${ }^{207}$.

2. Advoc.: - Fundado por Pere Doriz o Durà en su testamento ${ }^{208}$. El 12 de septiembre de 1338, vacante tras la muerte de Joan de Pulla, es concedido a Jaume Arbocer ${ }^{209}$. El 17 de julio de 1346 pasa a Jaume de Cursa, tras el fallecimiento del mentado Jaume Arbocer ${ }^{210}$.

3. Advoc.: - Fundado por Oria, mujer de Tomás de Próxida en 1309. El 6 de octubre de 1340 es concedido a Pere Mallol ${ }^{211}$.

\section{De Santa María de Calatrava}

1. Advoc.: - Fundado por Peregrí, alcaide del castillo de Bétera. El 31 de octubre de 1342, vacante tras la muerte del beneficiado, es colado a Guillem Tomás ${ }^{212}$.

2. Advoc.: - Fundado por Ferran Díez, también llamado Ferran de Nandum Diez. El 3 de junio de 1329, tras la muerte de Llorenç Guitart, es concedido a M. Tortajada ${ }^{213}$. El 4 de mayo de 1337, es conferido a

\footnotetext{
${ }^{20.3}$ AMV, Secc. I, Fondo III, carpeta 1, fol. 17.

${ }^{204}$ AMV, Secc. I, Fondo III, carpeta 1, fol. 7

${ }^{205}$ Funda seis capellanías que en 1343 pasarán a cinco. Cfr: AMV. Secc. I. Fondo III. carpeta 1. foll. 34.

${ }^{206} \mathrm{AMV}$, Secc. I, Fondo III. carpeta 1, fol. 30v

${ }^{207}$ AMV. Secc. I, Fondo III. carpeta 1, fol. 4v.

${ }^{208}$ En la documentación alterna el uso de los dos apellidos.

${ }^{209} \mathrm{AMV}$, Secc. I, Fondo III, carpeta 1, fol. 12

${ }^{210}$ AMV, Secc. I, Fondo III, carpeta 1, fol. 54v

2"AMV, Secc. I, Fondo III. carpeta 1, fol. 27v.

2'AMV, Secc. I, Fondo III, carpeta 1, fol. 31.

${ }^{213} \mathrm{AMV}$, Secc. I, Fondo III, carpeta 1, fol. 3v.
} 
Guillem de Saranyano al quedar vacante tras la muerte de Romeu Martínez $z^{214}$, acaecida el 2 de abril de $1337^{215}$.

De la Trinidad (Orden de San Francisco)

1. Advoc.: Santa María Virgen. Fundado por Ramón Compte. El 10 de noviembre de 1342 es concedido a Jaume de Sant Vicent ${ }^{216}$.

De Santa María de Montesa (Orden del Temple)

1. Advoc.: San Gregorio. Fundado por Guillem Ça Noguera. El 11 de diciembre de 1337 es concedido a Nicolau Piera ${ }^{217}$. El 10 de marzo de 1346 es conferido a Nicolau Piera, vacante tras la muerte de Joan Pujades. El mismo día se produce la presenación de Sanç Martí, con motivo de la renuncia del recién nombrado Nicolau Piera ${ }^{218}$.

\section{De la Trinidad (Orden de San Francisco)}

1. Fundado por Guillem Escrivà. El 17 de septiembre de 1334 es colado a fra Aparici Roca ${ }^{219}$.

\section{De la Zaidia (Orden del Císter)}

1. Advoc.: San Miguel Arcángel. Fundado por Francesca Pere de Montcada el 31 de julio de 1339. El 26 de agosto del mismo año es concedido a Martí Colom ${ }^{220}$.

2. Advoc.: Todos los Santos. Fundado por Beatriu de Llúria. El 8 de noviembre de 1336 es concedido a Bertomeu Urdells ${ }^{221}$.

3. Advoc.: - Fundado por Miquel García de Lunberri. El 8 de agosto de 1335 es concedido a Ferran Bosano ${ }^{222}$.

\footnotetext{
${ }^{214}$ AMV, Secc. I, Fondo III, carpeta 1, fol. 8.

${ }^{215}$ AMV. Secc. I, Fondo III, carpeta 1. fol. 7v.

$216 \mathrm{AMV}$, Secc. I, Fondo III, carpeta 1, fol. 31.

${ }^{217} \mathrm{AMV}$, Secc. I, Fondo III, carpeta 1, fol. 11.

${ }^{218} \mathrm{AMV}$, Secc. I, Fondo III, carpeta 1, fol. 42v.

${ }^{219} \mathrm{AMV}$, Secc. I, Fondo III, carpeta 1, fol. 15v.

${ }^{220}$ AMV, Secc. I, Fondo III, carpeta 1, fol. 6.

${ }_{221}$ AMV, Secc. I, Fondo III, carpeta 1, fol. 18v.

22 AMV. Secc. I, Fondo III, carpeta 1, fol. 15v.
} 
4. Advoc.: - Fundado por Sanç Pere de Rivabellosa, alcalde de Jérica, el 16 de agosto de 1329223. El 3 de noviembre de 1339 , vacante tras la muerte de Guillem Bondia, es concedido a Francesc Guerau ${ }^{2-4}$. El 18 de febrero de 1342 se ordena la toma de posesión de un nuevo beneficiado: Francesc Queralt ${ }^{225}$.

5. Advoc.: - Fundado por Bertomeu de Pont. El 3 de noviembre de 1329, vacante tras la muerte de Domènec de Monostrano, es concedido a Eximén Pere Navarro ${ }^{226}$.

6. Advoc.: - Fundado por el conde de Terranova ${ }^{227}$.

7. Advoc.: - Fundado por Miquel Llop de Rufes y su mujer Sança el 17 de enero de $1317^{228}$. El 5 de febrero de 1317 es concedido a Jaume Mitjavila229.

8. Advoc.: - Fundado por Alfons Xova, cavallero. El 30 de mayo de 1317 es concedido a Joan Gascó con motivo de la permuta habida con Enneco Sanç ${ }^{23.1}$.

9. Advoc.: - Fundado por Teresa Eiximemis, mujer de Alfons Xova. En 1334, vacante tras la muerte de su beneficiado es colado a Pere Gonçal ${ }^{231}$.

\section{D) Hospitales de la ciudad}

Hospital d'En Clapers

1. Advoc.: - Fundado por Bernat d'En Clapers. En 1317 es concedido a Ramón Tintorer ${ }^{232}$.

\footnotetext{
${ }^{223}$ AMV. Secc. I, Fondo III. carpeta 1. fol. 3v.

${ }^{22}$ AMV. Secc. I, Fondo III, carpeta 1. fol. 6v.

${ }^{225}$ AMV. Secc. I. Fondo III. carpeta 1, fol. 25v

"26AMV. Secc. I, Fondo III, carpeta 1, fol. 4.

${ }^{227}$ AMV. Secc. I, Fondo III, carpeta 1, fol. $11 \mathrm{v}$

$2:$ AMV. Secc. I, Fondo III, carpeta 1, fol. Iv.

2"AMV. Secc. I, Fondo III, carpeta 1, fol. 2.

${ }^{230}$ AMV. Secc. I, Fondo III, carpeta 1, fol. 2v.

${ }^{231}$ AMV, Secc. I, Fondo III, carpeta 1, fol. 14.

${ }^{232}$ AMV, Secc. I, Fondo III, carpeta 1, fol. 2.
} 
Hospital de Sant Guillem (Orden de la Trinidad)

1. Advoc.: - Fundado por Guillem Escrivà ${ }^{233}$.

\section{Hospital de San Lázaro}

1. Advoc.: Jesucristo y San Lázaro. Fundado por Bernat de Narbona y su mujer Estefanía en 1263. El 22 de septiembre de 1340 es concedido a Bernat de Mas, vacante tras la muerte de Guillem de Maraça ${ }^{234}$.

\section{RÉSUMÉ}

Dans ce travail on analyse l'organization de cette institution avec l'indication de leur localisation dans la ville de Valencia, ses advocations, ses fondateurs et ceux qui l'ont joui.

\section{SUMMARY}

This paper describes all the benefices in the city of Valencia, together with their dedication, founders and those who were engaged with them.

${ }^{233}$ AMV, Secc. I, Fondo III, carpeta 1, fol. 32.

${ }^{23.4}$ AMV. Secc. I, Fondo III, carpeta 1, fol. 22. 